\title{
A Combined Expectation Maximization and Marker Controlled Watershed Driven Distance Regularized Level Sets for Nuclear Segmentation in Histopathological Images
}

\author{
P. M. Shivamurthy \\ Sri Jayachamarajendra College of Engineering \\ Mysuru, India
}

\author{
T. N. Nagabhushan \\ Sri Jayachamarajendra College of Engineering \\ Mysuru, India
}

\author{
Vijaya Basavaraj \\ JSS Medical College and Hospital \\ Mysuru, India
}

\begin{abstract}
Detection of significant biomarker has been a major issue in the pathological study of cancerous tissue. The microscopic observation of H\&E stained histopathological slides involves the study of various tissue objects and their significant traits influencing the cancer grading. The change in shape and size of the nuclei objects (nuclear pleomorphism) contributes much significantly in grading the cancer. With the advent of various imaging studies over a digitized Histopathological image, Active contour based segmentation approaches are considered to be much potential scheme in detecting the nuclei within occlusions and extracting their irregular boundaries. In this research, a novel approach of driving the contours of distance regularized level sets using an improved watershed transformation, has been presented. An Expectation Maximization based morphologically precomputed shape prior is used to extract the foreground markers, which controls the watershed transformation. The result of watershed transformation is used compute the centroid of nuclei to serve the initialization of the contour and the proposed gradient to drive the same efficiently. The study performed over the Benign and Malignant tissue images from BreakHis dataset has shown the efficacy of the methodology in terms of object detection accuracy and overlap resolution. The segmentation accuracy is compared to that of Geodesic active contours, based on the ground truth generated by expert pathologist.
\end{abstract}

\section{General Terms}

Cancer grading, Nuclear Detection, Boundary Extraction

\section{Keywords}

Nuclear pleomorphism, Centroid Detection, Watershed gradient, Contour Evolution

\section{INTRODUCTION}

The advent digital pathology has immensely motivated the significant explorations in pathological studies involved in disease diagnosis and prognosis. Cancer grading is one such unsolved and challenging issue, which has resulted in many interesting findings through digital histopathology and its study involving various image processing approaches. Among the many biomarkers used in grading, nuclear pleomorphism is an important geometric feature which indicates the change in shape and size of the nuclei objects in the tissue. The rate of pleomorphism helps in exploring the progression of cancer through relative grading. Since the study of nuclear pleomorphism in a H\&E stained slide at microscopic level is a laborious process, it often leads to wrong diagnosis due to its complex morphological structure and also due to variabilities during the microscopic observation over the entire slide. Major task is to manually delineate the boundary of nuclei objects and segment out them for further studies. Due to high irregularity and missing boundary information the process of delineation is highly challenging and overlapped nuclei objects further enhances the complexity of the process.

Digitization of the histopathological slides have reduced the risk of misdiagnosis caused due to variabilities in the microscopic observation. A digitized image is obtained at different zooming level considering the pixel intensities as the source of data over a 2-D scene defined as $I=(P, \psi(x, y))$, where $P$ represents the matrix of pixels corresponding to RGB components and $\psi(x, y)$ is the function of pixel intensities $w \in P$. $w$ is a vector representing intensity levels of red, green, and blue channels of each pixel. Various computational transformations over pixel elements provides enough scope for detection of objects and extraction of the boundary.

\section{EARLIER WORK}

Earlier works proposed in many literature have emphasized two major aspects of extracting the nuclear objects. First, the object de- 
tection in a overlap resolution and the second, segmentation of the irregular boundary with missing information. To achieve these objectives, significance of various features and similarity measures have been presented in most of the earlier works. Segmentation based on a similarity measure computed using object based texture analysis was proposed by [29]. Cooccurence similarity among the tissue objects was adapted as an useful measure of object extraction by [25, 28]. The method proposed by [21] has adapted various structural features to segment the tissue objects. In the work presented by [5, 31], a combination of intensity, gradient and shape features have been adapted to drive the watershed segmentation towards the extraction of regions of interest. Integration of low, high and domain specific features was found to be useful combination to extract the object of interest in [19 20]. Most of the features extracted were either based on edge information or region information. A detailed summary on histopathological imaging techniques adapting both the set of features have been presented and a highlight on their deficiencies have been made in [9, 12]. It has been summarized that edge based methods lack in efficiency due to irregular and discontinuous boundary information and the same with region based approaches which results in over/under segmentation. Hence, most of the literature emphasizes on the need for an high level approach addressing both the objectives listed earlier.

\section{SYSTEM OVERVIEW}

The research work proposed here has been able to detect the object under overlapped resolution and also extract the boundary information of the nuclei effectively from a 2-D digitized image grid $P$. The following section provides the description of dataset used in this research.

\subsection{Dataset and Notations}

The dataset used is a collection of digitized images extracted from the sections of H\&E stained histopathological slides of beast cancer tissue sample. The source of dataset is from the collection provided by [27] and few live samples obtained from the pathologists involved in this research. It has been indicated in [27] that the collection is an extract from the samples obtained from surgical open biopsy(SOB) of both benign and malignant samples with different clinical representation. A total of 200 images extracted from tissue samples showing adenosis of benign and ductal carninoma of malignant have been chosen for experimentation. The resolution of images are chosen at a zooming level of 400x.

It is observed from the images shown in Figure 1 that the actual challenge for the pathologists lies in identifying the occluded objects and extracting the shape information by delineating the boundary of each objects. The proposed research has been able to achieve this by considering the 2-D image grid representation $P(x, y)$ of the image scene $I$ at a resolution of 700X 460 and performing marker controlled watershed transformation $W_{t}$ to generate the foreground regions. The marker controlled foreground regions thus obtained serves two purposes. First, the detection of centroids $C_{o}$, which provides the count of objects present and second,the watershed gradient $I_{g}$ computed on the foreground region serves as the driving force for the active contour initialized at the centroid towards extraction of the object boundary.Throughout this research various notations are used as listed in Table. 1

\subsection{The Phases of proposed method}

There are two major phases of the proposed research. First, detection of object centroid to obtain the actual count of nuclei objects in an occluded region. Second, segmentation of the irregular boundary of each detected nuclei using a deformable model which is initialized at the centroid detected for each object. Figure 2 shows the flow diagram depicting the overview of the entire procedure.

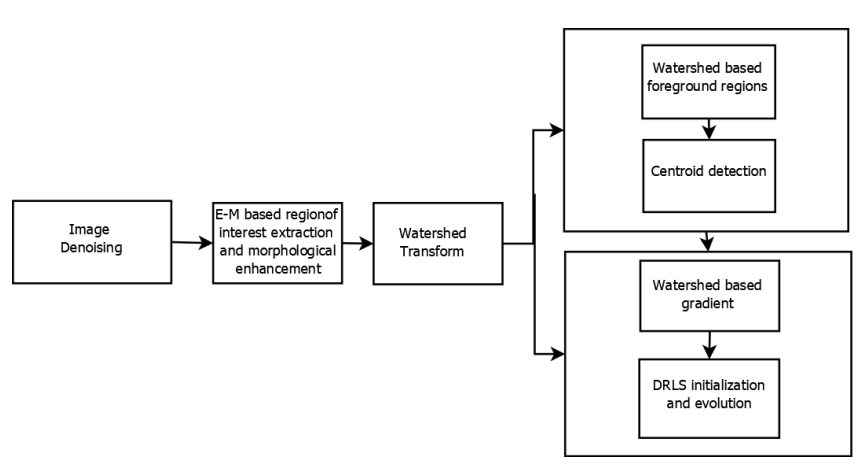

Fig. 2. Flow diagram showing the various phases of the proposed method.

A brief overview of these phases are given in the following sections

\subsection{Centroid detection}

Detecting centroids of each object is an important task in providing the count of nuclei in a overlapped region. This also serves as the seed point for the initialization of the contour evolution to further assist the segmentation of the object boundary. It is the geometrical center of the object at the position $x_{c}$ and $y_{c}$ in the 2-D image grid $P$. Centroids of all the objects in a occluded region is generated as $C_{o}=\left\{c_{i}=P\left(x_{i}, y_{i}\right) \in I\right\}$. A review on various centroid detection schemes based on Euclidean distance map, Hough transform, and H-maxima transform has been made in [13, 32]. A ground truth based seed point detection methods have been presented in [30, 10] considering nuclei size as the biomarker. A centroid prediction based on support vector machine(SVM) has been proposed by [17] and a deep learning based approach has been presented in [26]. All these methods employ a complex scheme of computation and hence necessitates a need for less complex method.

\subsection{Contour Evolution}

Ever since the idea proposed by [14], the active contours serves as the most promising energy minimizing deformable models to segment the objects with irregular boundaries. Hence, it is able to address the deficiencies of most of the edge and region based segmentation approaches. The energy is represented as the sum of the gradient information of the boundary region $E_{I}$ and the energy of the contour $E_{u}$. The contour $u$, is a polynomial, whose evolution towards the object boundary is controlled by the gradient information derived out of $E_{I}$ and $E_{u}$. This energy diminishes gradually towards null value as the contour evolve towards the object boundary. The contour is modeled with a partial differential equation represented by a level set function $\phi(u)$ as shown in Eq. 1

$$
\phi(u)=\frac{\partial u}{\partial t}+f(|\nabla u|,|\nabla I|)=0,
$$




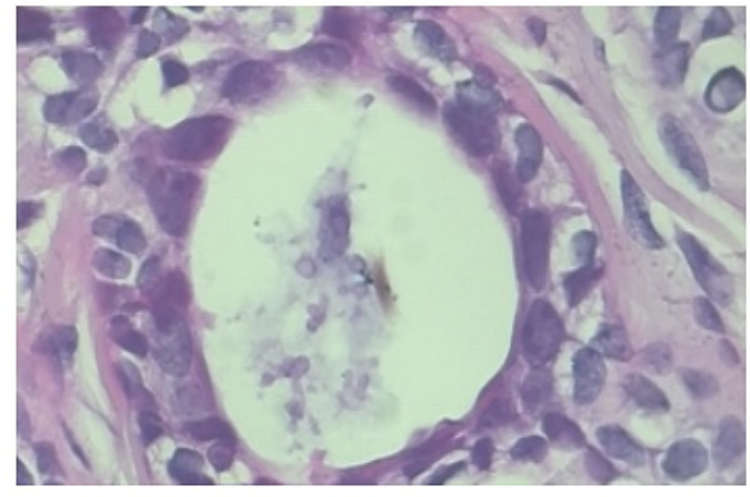

(a) Benign Tissue sample (Adenosis)

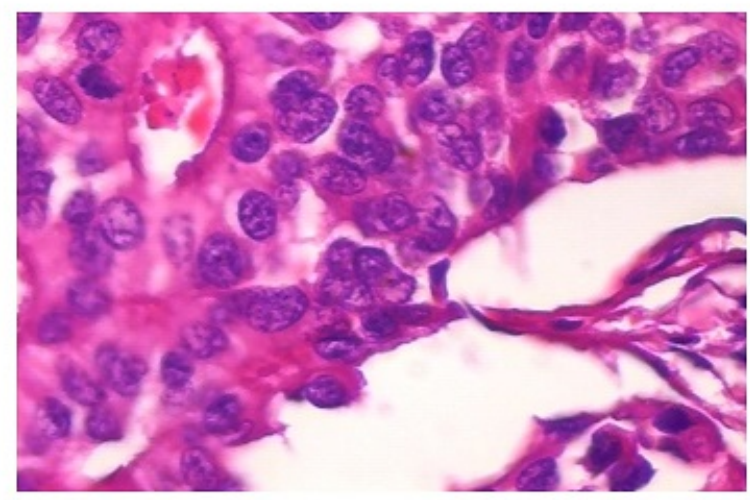

(b) Malignant Tissue sample (Ductal Carcinoma)

Fig. 1. H\&E stained image samples of both benign and malignant tissue at $400 \mathrm{X}$ zoom level.

Table 1. List of symbols used in this research

\begin{tabular}{|c|c||c|c|}
\hline Symbol & Description & Symbol & Description \\
\hline \hline$I$ & 2-D Image scene & $W_{t}$ & Watershed transform \\
\hline$I_{g}$ & Watershed gradient & $I_{f}$ & foreground image \\
\hline$P$ & 2-D image grid & $w$ & Intensity value of each pixel component \\
\hline$C_{o}$ & Set of centroids & $c_{i}$ & centroid of each object \\
\hline$T_{d}$ & topographical distance & $d_{p v}$ & Diffusion rate \\
\hline$F_{m}$ & Foreground marker & $\varepsilon_{e x t}$ & External energy gradient \\
\hline$\psi$ & Intesity function & $\nabla$ & gradient function \\
\hline$E_{I}$ & Energy gradient of Image & $E_{u}$ & Energy gradient of contour \\
\hline$u$ & contour represntation & $\phi$ & level set function of the contour \\
\hline$\alpha$ & Image signal component & $T_{k}$ & clusters of tissue regions \\
\hline$P P$ & Posterior probability & $p_{i}$ & prior probability \\
\hline$C_{b}$ & Catchment basin of watershed & $p v$ & potential value \\
\hline$\partial$ & Partial differential & $f$ & distance function \\
\hline
\end{tabular}

where $f$ is the function which computes the edge or region based gradient information using $\nabla$, depending on the approach of contour evolution. Most of the previous works on active contours have laid a major emphasis on gradient computation based on the shape features. A multiple level set implementation based on both edge and region gradient has been presented by[2]. An active shape model was proposed by [1] to segment overlapped objects, which is based on a statistical model constrained by point distribution proposed by [7]. A novel approach of integrating shape, region, and boundary features and an adaptive energy computation, were presented by [3] and [4] respectively. Though, the edge based approaches have their own limitations of initialization and gradient computations, the Geodesic active contour presented by [6] is quite promising, as shown in [11]. An improved approach based on Distance Regularized Active Contour (DRLS), was proposed by [15]. Another approach, which combines both the edge and region gradient information for evolving the contours was presented by [8]. The energy minimizing model presented by [18] is adapted to compute the gradient for the same.

In this research, the DRLS based active contour model presented by [15] is adapted for an efficient segmentation of the objects. This model is controlled by a distance regularization term, which works based on forward and backward diffusion effects during evolution. This reduces the reinitialization at every iterations and hence results in fewer iterations and lesser reinitialization errors. The mathematical model is as shown in Eq. 2

$$
\frac{\partial \phi}{\partial t}=\mu \operatorname{div}\left(d_{p v}(\nabla \phi) \nabla \phi\right)-\frac{\partial \varepsilon_{e x t}}{\partial \phi}
$$

The first term is the distance regularization term as shown in Eq. 3

$$
\frac{\partial \phi}{\partial t}=\mu \operatorname{div}\left(d_{p v}(\nabla \phi) \nabla \phi\right)
$$


The term $d_{p v}=\mu d_{p v}(\nabla \phi)$, is the diffusion rate, which takes either a positive or negative potential value $p v$. The positive and negative values represent forward and backward diffusion respectively. The second term is the derivative of the external energy functional $\varepsilon_{\text {ext }}$ with respect to $\phi$.

The efficacy of the DRLS technique has been exploited by using an improved method of gradient computation and the centroid based contour initialization. The basic idea is to obtain the watershed transform $W_{t}$ of the original RGB image grid $P$, which is controlled by the foreground markers $F_{m}$ generated using the precomputed shape prior. The foreground scene $I_{f}$ is obtained by applying expectation-maximization on the enhanced original image. The transform $W_{t}$ can be used for finding out the object centroids $C_{o}$ considering the watershed regions corresponding to the foreground markers $F_{m}$ as well as to compute the energy gradient $I_{g}$ that corresponds to, $\varepsilon_{\text {ext }}$, the external energy component of the DRLS. The following section presents a detailed algorithm description of the proposed research. Further, Sections 4 and 5 discuss the experimentation and result analysis respectively, followed by conclusion.

\section{THE PROCEDURE}

In this section, the various phases of the proposed methodology discussed in the previous section are elaborated in detail. An algorithmic illustration of each stages has been presented in Algorithm 1.

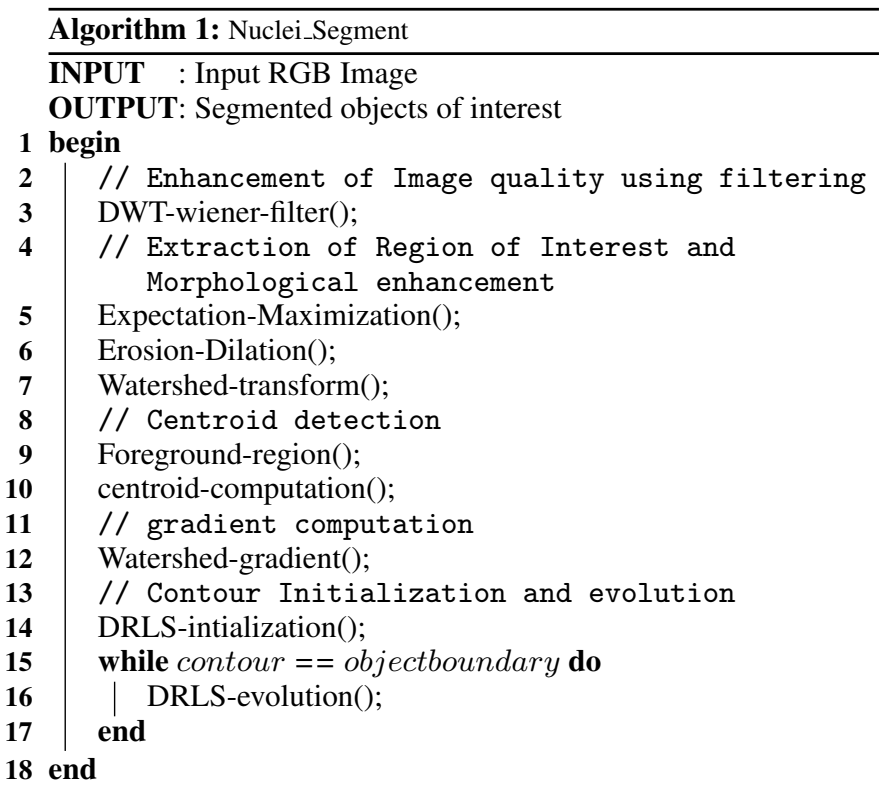

\subsection{Image denoising}

It is evident from the fact that, due to staining errors and also due to the zooming errors, the digitized 2-D image $P(x, y)$ of the $\mathrm{H} \$ \mathrm{E}$ stained slide suffers from both high and low frequency noise components. Hence, both the components has to be separated to perform filtering. The discrete wavelet transform (DWT) of the image splits the signal into different frequency sub bands at various spatial resolutions. Both low and high frequency components represented as $\alpha_{\text {low }}$ and $\alpha_{\text {high }}$ respectively are computed as shown in Eq. 4 and Eq. 5

$$
\begin{gathered}
\alpha_{\text {low }}(n)=\sum_{i=-\infty}^{\infty} \alpha(i) \operatorname{lp}(2 n-i) \\
\alpha_{\text {high }}(n)=\sum_{i=-\infty}^{\infty} \alpha(i) h p(2 n-i),
\end{gathered}
$$

where $\alpha(i)$ is the signal component and the functions $l p$ and $h p$ are the low and high pass filter functions respectively. The significant role of DWT in reducing the over segmentation is shown in [22]. In a work presented by [24], it has been shown that Wiener filer integrated with DWT performs effective filtering of both the noise components. Using this idea, the denoised signal coefficient can be computed as shown in Eq. 6

$$
D(i, j)=\mu+\frac{\sigma^{2}-N}{\sigma^{2}}(s(i, j)-\mu)
$$

$N$ is the noise component and the parameters $\mu$ and $\sigma^{2}$ are the mean and variance respectively, as shown in Eqs.7 7 and 8

$$
\begin{gathered}
\mu=\frac{1}{n^{2}} \sum_{i=1}^{n} \sum_{j=1}^{n} s(i, j) \\
\sigma^{2}=\frac{1}{n^{2}} \sum_{i=1}^{n} \sum_{j=1}^{n} s^{2}(i, j)-\mu^{2},
\end{gathered}
$$

where $s(i, j)$ is the sub-band generated by DWT.

\subsection{Expectation-Maximization and Watershed transformation}

The next task is to obtain the region of interest from the denoised image with a suitable clustering approach. Keeping the four different tissue components viz., nuclei, stroma, cytoplasm and gland sections, as the reference, a set of four clusters of image pixels, $T_{k}$ is generated using Expectation-maximization techniques. Initially, the E-step computes the posterior probabilities $P P\left(T_{k} \mid f(c)\right)$ of pixel components from each class in $T_{k}$ for the $k$ values ranging from 1 to 4 . The prior probabilities for the same is obtained taking Gaussian distribution of four randomly chosen classes. In the M-step, the model parameters mean,covariance and mixture coefficients denoted as $\gamma_{k}^{i}=\left\{\mu_{k}^{i}, \sigma_{k}^{i}, p_{k}^{i}\right\}$ are updated iteratively. The computation of posterior probabilities is given by Eq. 9

$$
P P\left(T_{k} \mid f(c)\right)=\frac{p_{k}^{i} \eta\left(f(c) \mid \mu_{k}^{i}, \Sigma_{k}^{i}\right)}{\Sigma_{j=1}^{K} p_{j}^{i} \eta\left(f(c) \mid \mu_{j}^{i}, \Sigma_{j}^{i}\right)}
$$

for all $c \in I(x, y)$ and $\eta$ is the $\mathrm{D}$-dimensional Gaussian distribution considering $\mathrm{D}$ as three for RGB dimensions as shown below.

$\eta\left(f(c) \mid \mu_{k}^{i}, \Sigma_{k}^{i}\right)=$

$(2 \pi)^{-D / 2}\left|\Sigma_{k}^{-1 / 2}\right| \exp \left\{-\frac{1}{2}\left(f(c)-\mu_{k}^{i}\right)^{T} \Sigma_{k}^{-} 1\left(f(c)-\mu_{k}^{i}\right)\right\}$

The rest of the computations of model coefficients of M-step and finally the convergence evaluation is performed as per the [11]. After obtaining the region of interest corresponding to nuclei objects $T_{k=n}$, where $n$ is the class corresponding to object of interest, the foreground markers $F_{m}$ are generated using morphological operations based on the shape model presented in [23]. These markers are going to drive the watershed transformation $W_{t}\left(T_{n}, F_{m}\right)$ of 
the regions of interest. The water source is flooded from a regional minima $m_{i}$ until the barriers are found using the floods of others sources. The catchment basin $C b_{m_{i}}$ of each regional minima is the set of all pixels points topographically closer to $m_{i}$ as shown Eq. 10

$$
\begin{aligned}
C b_{m_{i}} & =\left\{x \in I: T_{d}\left(x, m_{i}\right)<T_{d}\left(x, m_{j}\right)\right\} \\
\forall \mathrm{j} & \in \mathrm{P} \backslash\{\mathrm{i}\}
\end{aligned}
$$

The $T_{d}$ is the topopraphical distance between two points $p$ and $q$ considering the steepest descent distance of the all the paths $\gamma(s)$ between those points as shown in Eq. 11 .

$$
T_{d}(p, q)=i n f_{\gamma} \int\left\|\nabla I_{c}(\gamma(s))\right\| d s
$$

Further, the watershed transform serves the dual purpose, first, to generate the region centroids using the foreground regions generated and second, to compute the gradient information of the regions as discussed in the following section.

\section{3 centroid detection and contour evolution}

In the last stage of the algorithm, the centroids of the regions, which are obtained using watershed transform, is computed and each centroid serves as the count for number of objects in the occluded region and also as the initialization point for the evolution of contour $\phi(u)$ towards segmentation of the object boundary. The DRLS contour evolution is controlled by the energy gradient information $I_{g}$ computed from the transform obtained in the previous section. It corresponds to $\varepsilon_{\text {ext }}$, which represents the external energy, whose derivative with respect to $\phi$ is the second term, which represents the external gradient term of the DRLS formulation as shown in section 3

\section{EXPERIMENTATION AND RESULTS}

The proposed method has been experimented over 200 images from the BreakHis data set discussed in section. 3. The resolution of the images selected here is 700X460 captured at 400x zoom level. Figure 3 shows the outcomes of various stages in the methodology. It is clearly evident from the results that the proposed method is able to extract the individual objects even if they are occluded. In the next section, a qualitative and quantitative analysis of the results by comparing it with the ground truth and with that of results obtained using Geodesic active contour respectively are presented.

\section{QUALITATIVE AND QUANTITATIVE ANALYSIS}

In this section, both qualitative and quantitative analysis of the results are performed. In Figure. 4 a comparison of the results obtained for few images using both DRLS with watershed initialization and without initialization is shown. The results show that the watershed transformation with foreground markers has been successful in extracting the overlapped objects with a higher degree as compared to that of without transformation.

According to [11], the following two types of quantitative measures are very suitable for the evaluation of the segmentation based on active contours. First, object detection and overlap metrics and, second, segmentation accuracy using boundary error metrics. The object detection measures are sensitivity (SN), specificity (SP), positive predictive value (PPV), and the overlap resolution (OR). These are computed using the direct measures extracted from the results, such as true positive (TP), true negative (TN), false positive (FP), and false negative (FN). The formulation are as given in [11].
As the task of ground truth generation performed manually by an expert pathologist is very tedious, only 30 selected images out of 200 images are chosen for quantitative analysis. The above listed measures are computed over the images chosen and the comparative results of both the approaches are presented in Table. 2. The

Table 2. Object Detection Measures for DRLS-WT and DRLS

\begin{tabular}{|c|c|c|c|c|}
\hline & SN & SP & PPV & OR \\
\hline DRLS & 0.91 & 0.83 & 0.90 & 0.86 \\
\hline DRLS-WT & 0.96 & 0.68 & 0.93 & 0.90 \\
\hline
\end{tabular}

other measures are the actual count (AC) of the number of nuclei and the overlaps present in the image, and the detected count (DC) obtained by the method proposed. The formulations of these measures are as presented in [11].

The above measures are computed by considering the average values derived out of 20 randomly chosen objects out of the sample images. A chart showing the AC and DC of both nuclei detection and overlap resolution is shown in Figure 5 The DC measures are computed and compared among both DRLS-WT and DRLS techniques.

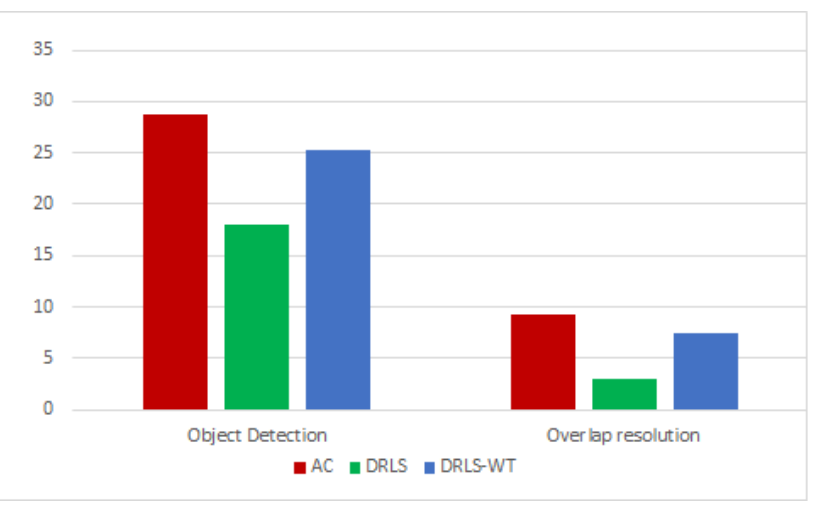

Fig. 5. Charts showing the comparative study of object detection and overlap resolution accuracy of DRLS and DRLS-WT with AC

This indicates that the DRSL-WT outperforms DRLS with respect to all the measures of object detection and overlap resolution.

Second, the segmentation accuracy is the measure of accuracy of extracting the nuclei boundary. They are measured using two metrics as proposed by [16]: (i). Hausdorff Distance (HD) and (ii). Mean Absolute Distance (MAD), as given in Eqs. 12 and 13

$$
\begin{gathered}
H D=\max _{\omega}\left[\min _{\chi}\left\|c_{\omega}-c_{\chi}\right\|\right] \\
M A D=\frac{1}{M} \sum_{\omega=1}^{M}\left[\min _{\chi}\left\|c_{\omega}-c_{\chi}\right\|\right]
\end{gathered}
$$

Since the manual delineation is a very tedious task, the pathologists have randomly chosen only 20 nuclei objects for generating ground truth. Considering the ground truth as reference, these measures are computed for the objects extracted using the proposed method. As a comparative study, the results are compared with that of geodesic 


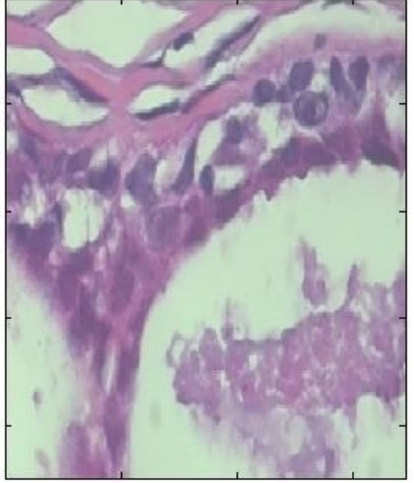

(a) Original Image

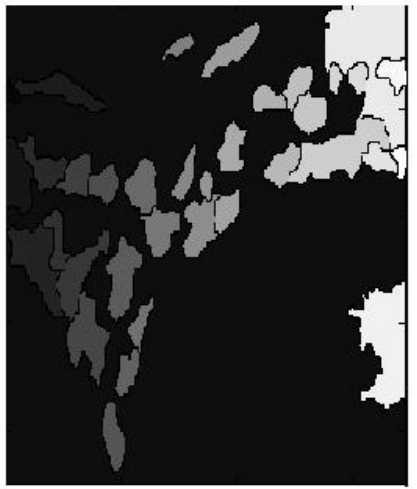

(d) Watershed gradient

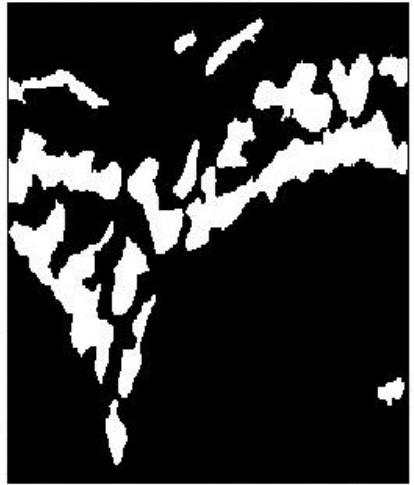

(b) Region of Interest using E-M

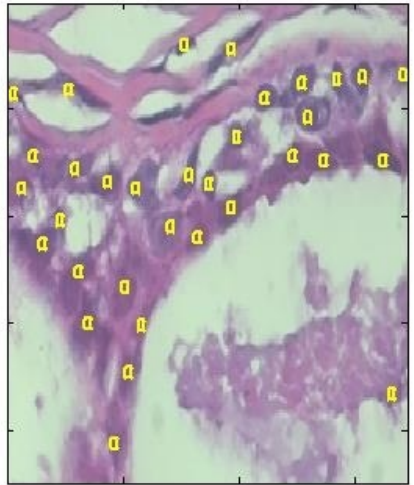

(e) Initial Contour

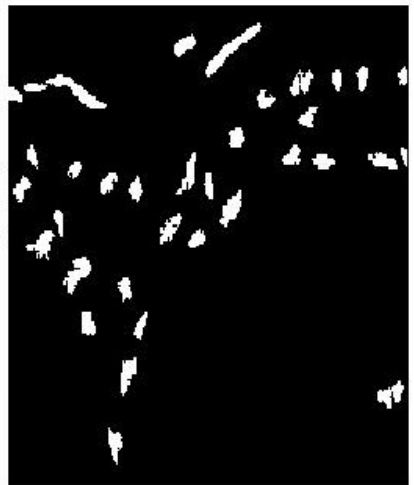

(c) Foreground Markers

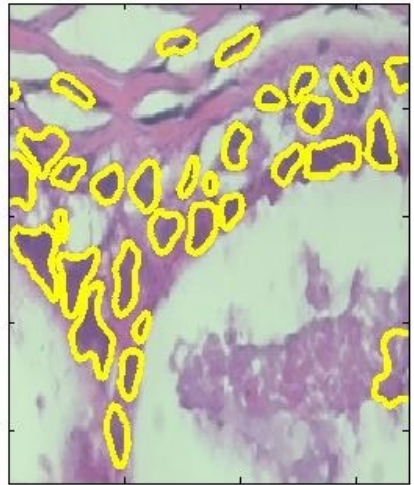

(f) Final Contour

Fig. 3. The result of each stage is shown in Figures (a)-(f)

active contour with watershed transforms (GAC-WT) as plotted in the charts shown in Fig. 6 and 7 The pixel difference between the contour and the manual delineation is the key factor during the computation of HD and MAD. The distance computed for DRLSWT has been measuring a very low pixel count of utmost 3 pixels, considering segmentation of all the objects. In contrast, the GACWT has the pixel difference ranging between 2 to 12 , demonstrating the efficiency of the proposed approach over the other.

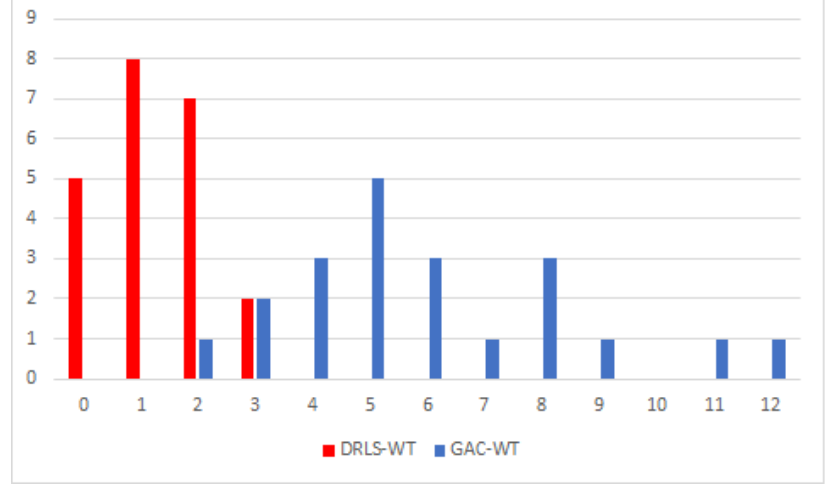

Fig. 6. Charts showing the Hausdorff distance (HD) comparison between DRLS-WT and GAC-WT with reference to ground truth 


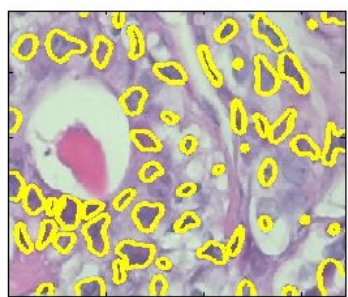

(a)

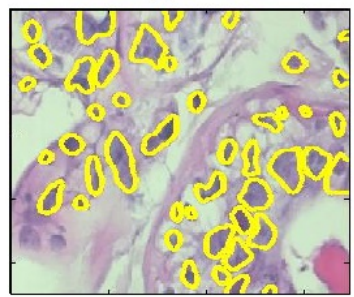

(b)
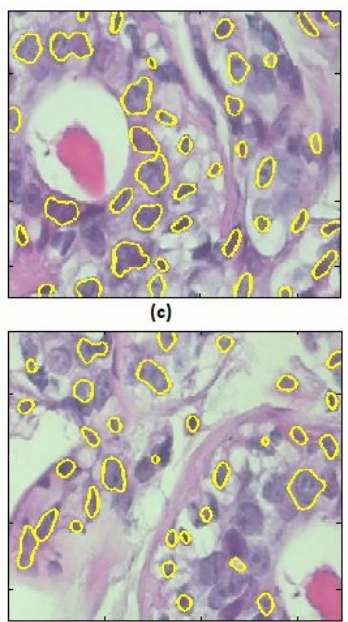

(d)
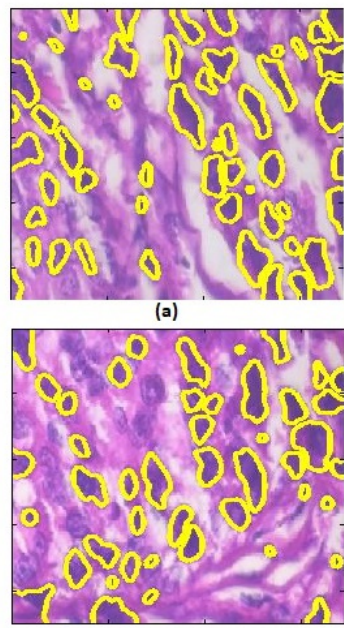

(b)
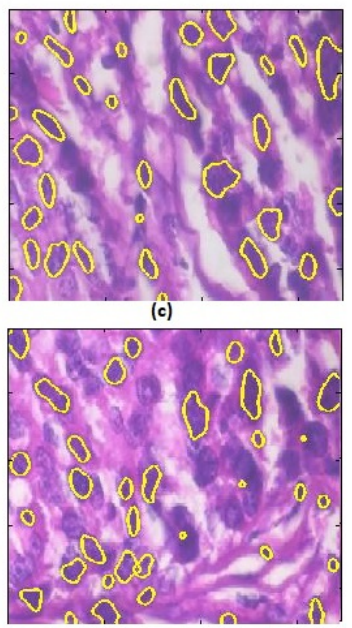

(d)

i. Benign Samples

ii. Malignant Samples

Fig. 4. (a) and (b) show the results with Watershed initialization (DRLS-WT) (c) and (d) show the results without Watershed initialization (DRLS)

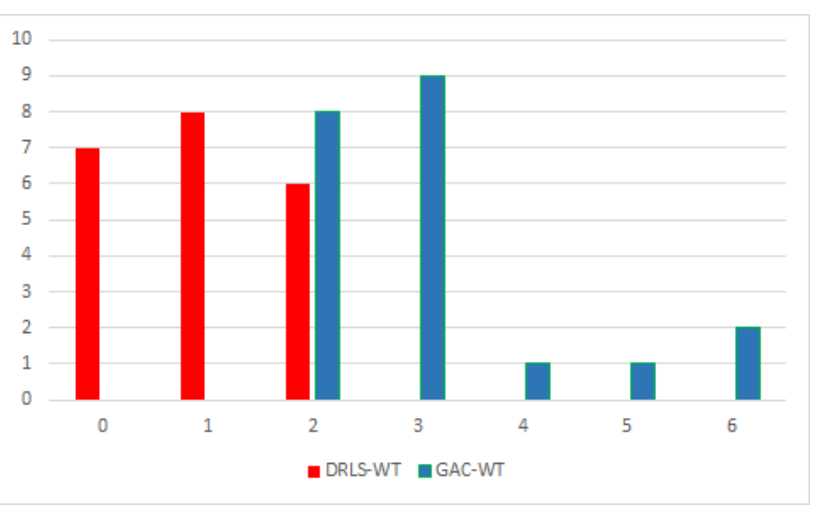

Fig. 7. Charts showing the Mean Absolute distance (MAD) comparison between DRLS-WT and GAC-WT with reference to ground truth

\section{CONCLUSION}

The challenging task of diagnosing and grading the cancer based on nuclear pleomorphism has been addressed effectively in this proposed research. The detection and segmentation of nuclei based DRLS, an edge based active contour, has been adapted to overcome most of the limitations faced by the pathologists. This has also been able to address various shortcomings of the existing low level approaches as presented in the literature survey. This method adapts a novel contour initialization and gradien computation techniques based on marker controlled watershed transformation. The methodology involves extraction of foreground regions from a watershed transform. This transformation is performed on the foreground marker obtained morphilogically using the regions of interest extracted by expectation maximization approach. These foreground regions assist in the computation of centroids of hidden nuclei in occluded region and computation of watershed gradient for effectively driving the DRLS towards the object boundary. The efficiency of the approach has been studied with respect to object detection and overlap resolution accuracy followed by the segmentation accuracy computed using boundary based metrics. The accuracy of the results have been presented in comparison with other techniques, keeping the ground truth generated by the expert pathologist as the reference. The results can be extended in future work to support further classification.

\section{REFERENCES}

[1] Sahirzeeshan Ali and Anant Madabhushi. Active contour for overlap resolution using watershed based initialization (acorew): Applications to histopathology. In Biomedical Imaging: From Nano to Macro, 2011 IEEE International Symposium on, pages 614-617. IEEE, 2011.

[2] Sahirzeeshan Ali and Anant Madabhushi. Segmenting multiple overlapping objects via a hybrid active contour model incorporating shape priors: applications to digital pathology. In SPIE Medical Imaging, pages 79622W-79622W. International Society for Optics and Photonics, 2011.

[3] Sahirzeeshan Ali and Anant Madabhushi. An integrated region-, boundary-, shape-based active contour for multiple object overlap resolution in histological imagery. IEEE transactions on medical imaging, 31(7):1448-1460, 2012.

[4] Sahirzeeshan Ali, Robert Veltri, Jonathan Epstein, Christhunesa Christudass, and Anant Madabhushi. Adaptive energy selective active contour with shape priors for nuclear segmentation and gleason grading of prostate cancer. Medical Image Computing and Computer-Assisted Intervention-MICCAI 2011, pages 661-669, 2011.

[5] Ewert Bengtsson, C Wahlby, and Joakim Lindblad. Robust cell image segmentation methods. Pattern Recognition and Image Analysis C/c of Raspoznavaniye Obrazov i Analiz Izobrazhenii., 14(2):157-167, 2004.

[6] Vicent Caselles, Ron Kimmel, and Guillermo Sapiro. Geodesic active contours. International journal of computer vision, 22(1):61-79, 1997. 
[7] Tony Chan and Wei Zhu. Level set based shape prior segmentation. In Computer Vision and Pattern Recognition, 2005. CVPR 2005. IEEE Computer Society Conference on, volume 2, pages 1164-1170. IEEE, 2005.

[8] Tony F Chan and Luminita A Vese. Active contours without edges. IEEE Transactions on image processing, 10(2):266$277,2001$.

[9] Cigdem Demir and Bülent Yener. Automated cancer diagnosis based on histopathological images: a systematic survey. Rensselaer Polytechnic Institute, Tech. Rep, 2005.

[10] Pegah Faridi, Habibollah Danyali, Mohammad Sadegh Helfroush, and Mojgan Akbarzadeh Jahromi. Cancerous nuclei detection and scoring in breast cancer histopathological images. arXiv preprint arXiv:1612.01237, 2016.

[11] Hussain Fatakdawala, Jun Xu, Ajay Basavanhally, Gyan Bhanot, Shridar Ganesan, Michael Feldman, John E Tomaszewski, and Anant Madabhushi. Expectationmaximization-driven geodesic active contour with overlap resolution (emagacor): Application to lymphocyte segmentation on breast cancer histopathology. IEEE Transactions on Biomedical Engineering, 57(7):1676-1689, 2010.

[12] Lei He, L Rodney Long, Sameer Antani, and George R Thoma. Histology image analysis for carcinoma detection and grading. Computer methods and programs in biomedicine, 107(3):538-556, 2012.

[13] Humayun Irshad, Antoine Veillard, Ludovic Roux, and Daniel Racoceanu. Methods for nuclei detection, segmentation, and classification in digital histopathology: a reviewcurrent status and future potential. IEEE reviews in biomedical engineering, 7:97-114, 2014.

[14] Michael Kass, Andrew Witkin, and Demetri Terzopoulos. Snakes: Active contour models. International journal of computer vision, 1(4):321-331, 1988.

[15] Chunming Li, Chenyang Xu, Changfeng Gui, and Martin D Fox. Distance regularized level set evolution and its application to image segmentation. IEEE Transactions on image processing, 19(12):3243-3254, 2010.

[16] Anant Madabhushi and Dimitris N Metaxas. Combining low, high-level and empirical domain knowledge for automated segmentation of ultrasonic breast lesions. IEEE transactions on medical imaging, 22(2):155-169, 2003.

[17] Antonio Morote. Nuclear detection in histopathology images. Thesis work, 2010.

[18] David Mumford and Jayant Shah. Optimal approximations by piecewise smooth functions and associated variational problems. Communications on pure and applied mathematics, 42(5):577-685, 1989.

[19] Shivang Naik, Scott Doyle, Shannon Agner, Anant Madabhushi, Michael Feldman, and John Tomaszewski. Automated gland and nuclei segmentation for grading of prostate and breast cancer histopathology. In Biomedical Imaging: From Nano to Macro, 2008. ISBI 2008. 5th IEEE International Symposium on, pages 284-287. IEEE, 2008.

[20] Shivang Naik, Scott Doyle, Michael Feldman, John Tomaszewski, and Anant Madabhushi. Gland segmentation and computerized gleason grading of prostate histology by integrating low-, high-level and domain specific information. In MIAAB workshop, pages 1-8. Citeseer, 2007.
[21] Kien Nguyen, Bikash Sabata, and Anil K Jain. Prostate cancer grading: Gland segmentation and structural features. Pattern Recognition Letters, 33(7):951-961, 2012.

[22] Aleš Procházka, Oldrich Vysata, and Eva Jerhotova. Wavelet use for reduction of watershed transform over-segmentation in biomedical images processing. In Information Technology and Applications in Biomedicine (ITAB), 2010 10th IEEE International Conference on, pages 1-4. IEEE, 2010.

[23] Nagabhushan T.N. Shivamurthy, P.M. and V. Basavaraj. A foreground marker based centroid initialized geodesic active contours for histopathological image segmentation. Cognitive Computing and Information Processing (CCIP), 2016 Second International Conference on. IEEE., pages 1-6, 2016.

[24] Mohammed M Siddeq and Dr Sadar Pirkhider Yaba. Using discrete wavelet transform and wiener filter for image denosing. Wasiit Journall for Sciience Mediiciine, 2(2):18-30, 2009.

[25] Ahmet Cagri Simsek, Akif Burak Tosun, Cevdet Aykanat, Cenk Sokmensuer, and Cigdem Gunduz-Demir. Multilevel segmentation of histopathological images using cooccurrence of tissue objects. IEEE Transactions on Biomedical engineering, 59(6):1681-1690, 2012.

[26] Korsuk Sirinukunwattana, Shan E Ahmed Raza, Yee-Wah Tsang, David RJ Snead, Ian A Cree, and Nasir M Rajpoot. Locality sensitive deep learning for detection and classification of nuclei in routine colon cancer histology images. IEEE transactions on medical imaging, 35(5):1196-1206, 2016.

[27] Fabio A Spanhol, Luiz S Oliveira, Caroline Petitjean, and Laurent Heutte. A dataset for breast cancer histopathological image classification. IEEE Transactions on Biomedical Engineering, 63(7):1455-1462, 2016.

[28] Akif Burak Tosun and Cigdem Gunduz-Demir. Graph runlength matrices for histopathological image segmentation. IEEE Transactions on Medical Imaging, 30(3):721-732, 2011.

[29] Akif Burak Tosun, Melih Kandemir, Cenk Sokmensuer, and Cigdem Gunduz-Demir. Object-oriented texture analysis for the unsupervised segmentation of biopsy images for cancer detection. Pattern Recognition, 42(6):1104-1112, 2009.

[30] JP Vink, MB Van Leeuwen, CHM Van Deurzen, and $\mathrm{G}$ De Haan. Efficient nucleus detector in histopathology images. Journal of microscopy, 249(2):124-135, 2013.

[31] Carolina Wählby, I-M SINTORN, Fredrik Erlandsson, Gunilla Borgefors, and Ewert Bengtsson. Combining intensity, edge and shape information for $2 \mathrm{~d}$ and $3 \mathrm{~d}$ segmentation of cell nuclei in tissue sections. Journal of Microscopy, 215(1):6776, 2004.

[32] Fuyong Xing and Lin Yang. Robust nucleus/cell detection and segmentation in digital pathology and microscopy images: a comprehensive review. IEEE reviews in biomedical engineering, 9:234-263, 2016. 